\title{
Combination therapy with BMP-2 and psoralen enhances fracture healing in ovariectomized mice
}

\author{
KUI HUANG ${ }^{1,2}$, GUOFENG WU ${ }^{1}$, JI ZOU ${ }^{2}$ and SONGMING PENG ${ }^{1}$ \\ ${ }^{1}$ Department of Orthopaedics, The First Affiliated Hospital of Yangtze University, Jingzhou, Hubei 434000; \\ ${ }^{2}$ Department of Acupuncture-Moxibustion and Orthopaedics-Traumatology, \\ Hubei University of Chinese Medicine, Wuhan, Hubei 430065, P.R. China
}

Received January 1, 2018; Accepted May 17, 2018

DOI: $10.3892 /$ etm.2018.6353

\begin{abstract}
The advantages of combining local delivery of bone morphogenetic protein (BMP)-2 with systemic or local anti-osteoporosis treatments have also been studied for enhancing osteoporotic fracture healing. The aim of the present study was to evaluate the effect of combination therapy with BMP-2 and psoralen on fracture repair in ovariectomized mice. At 6 weeks after bilateral ovariectomy, mice $(n=30)$ underwent unilateral transverse osteotomy on the femur and were divided into 3 groups. In the model group $(n=10)$, animals were implanted with an absorbable collagen sponge (ACS) alone and administered physiological saline intragastrically (i.g.). In the recombinant human (rh)BMP-2 group $(n=10)$, animals were implanted with an ACS loaded with $2.5 \mu \mathrm{g}$ rhBMP-2 and administered physiological saline i.g. In the psoralen + rhBMP-2 group $(n=10)$ animals were implanted with an ACS loaded with $2.5 \mu \mathrm{g}$ rhBMP-2 and administered psoralen i.g. The mice were euthanized after 21 days and their fractured femurs were assessed by micro computed tomography, histological analysis and biomechanical testing. Furthermore, the serum of the animals was analyzed. Psoralen + rhBMP-2 exerted more beneficial effects on callus consolidation and biomechanical strength. In addition, increased bone-specific alkaline phosphatase levels and decreased C-terminal telopeptide of type-1 collagen were observed in the Psoralen + rhBMP-2 group. However, no difference in estrogen levels was detected between the groups. In conclusion, the present study demonstrated that in
\end{abstract}

Correspondence to: Professor Ji Zou, Department of Acupuncture-Moxibustion and Orthopaedics-Traumatology, Hubei University of Chinese Medicine, 1 West Road, Wuhan, Hubei 430065, P.R. China

E-mail: dr_zj@126.com

Mr. Songming Peng, Department of Orthopaedics, The First Affiliated Hospital of Yangtze University, 8 Hangkong Road, Jingzhou, Hubei 434000, P.R. China

E-mail: psmingdr@163.com

Key words: recombinant human bone morphogenetic protein-2, fracture healing, osteoporosis, phytoestrogens ovariectomized mice, combination of locally delivered BMP-2 and systemically administered psoralen improved bone healing compared with BMP-2 alone.

\section{Introduction}

Osteoporosis is a major public health problem due to its association with bone fragility and fracture, particularly as the aging population has grown in recent years. Osteoporosis is caused by an imbalance between bone resorption and formation, which leads to a reduction in osteogenic ability as well as to excessive bone resorption. These adverse processes may result in delayed bone healing or nonunion of fractures (1-3). The identification of osteopromotive and inductive agents with the potential to stimulate bone formation has raised hope for the augmentation of osteoporotic fracture healing (4,5). Local delivery of recombinant human bone morphogenetic protein (rhBMP-2) has been approved by the U.S. Food and Drug Administration for the promotion of spinal fusion and fracture healing (6). In in vivo studies, local application of BMP-2 in animal models of osteoporotic bone fracture was demonstrated to be a promising therapeutic approach (7-9). In spite of the success of its clinical application $(10,11)$, administration of BMP-2 has a number of drawbacks. The optimum release pattern of rhBMP-2 has remained to be established, and excessive dosages of BMP may be dangerous, as they have other effects in processes including organogenesis, cell differentiation, cell proliferation and apoptosis. Furthermore, the production and purification of the recombinant protein is costly, making this therapy expensive (12-14).

Considering that the side effects of rhBMP-2 are dose-dependent, it may be reasonable to reduce the large doses of BMP-2 that are currently used. One possible alternative to overcome this problem is synchronous drug combinations to enhance the potency of the BMP-2 used. The combination of systemic or local anti-osteoporosis treatments with local delivery of BMP-2 has also been studied for enhancing bone formation $(15,16)$. As osteoporosis is a systemic skeletal disease, appropriate simultaneous management of the osteoporotic fracture and osteoporosis is adequate and essential.

The current clinical treatment regimens for osteoporosis are anti-resorptive drugs, including estrogen, estrogen receptor analogues, calcitonin and bisphosphates, which maintain 
the bone mass by inhibiting osteoclast function. However, studies on the negative effects of fracture healing treatments and potential complications, including breast cancer, uterine bleeding and cardiovascular events, have raised concerns regarding their long-term use $(17,18)$.

Chinese herbal medicines have been widely used to prevent and treat diseases for thousands of years. Psoralen (molecular structure displayed in Fig. 1A) is a coumarin derivative extracted from the dried fruit of Psoralen Corylifolia L., which is a well-known medicine with ascribed properties of 'bone strengthening' based on The Traditional Chinese Medicine theory. In an ex vivo study, psoralen increased the osteogenic potential of bone marrow mesenchymal stem cells (bMSCs), and stimulated the differentiation of bMSCs to osteoblasts, with enhanced alkaline phosphatase (ALP) activity (19). It has been reported that psoralen promotes bone mass formation, increases bone strength and improves the trabecular bone microstructure in ovariectomized (OVX) mice, suggesting that it may be used as a natural compound for the treatment of osteoporosis (20).

The aim of the present study was to evaluate the effects of combination therapy with BMP-2 and psoralen on fracture repair in OVX mice. Serum analyses of substances including bone-specific ALP (BALP) and C-terminal telopeptide of type-1 collagen (CTX-1) were also performed to study bone metabolism.

\section{Materials and methods}

Animals. In total, 40 female C57BL/6 mice (body weight, $20.7 \pm 1.3 \mathrm{~g}$; age, 12 weeks) were purchased from the Hubei Research Center of Laboratory Animal (Wuhan, China). They were housed and acclimatized at the experimental animal laboratory of Yangtze University (Jingzhou, China) under controlled temperature $\left(25^{\circ} \mathrm{C}\right)$ and humidity $(55 \%)$ with a $12-\mathrm{h}$ light/dark cycle, with free access to food and water.

Establishment of osteoporotic model. Female mice $(\mathrm{n}=35)$ were subjected to OVX and those in the sham group $(n=5)$ received a sham surgery. After 6 weeks, the establishment of the standard osteoporotic animal models was confirmed prior to bone fracture surgery. To verify the success of OVX, 5 randomly selected OVX mice and the 5 sham-operated mice were euthanized to measure the estrogen levels, and the femoral metaphysis was harvested for histological evaluation.

Study design. All OVX animals were randomly stratified into one of the following groups: Model group, rhBMP-2 group and Psoralen + rhBMP-2 group (Table I). According to previous studies, fracture bridging appeared to occur at 21 days after the fracture (21). Therefore, the mice of the present study were sacrificed on day 21 after injury and their limbs and blood were harvested for further analysis [micro computed tomography $(\mathrm{CT})$, histological, mechanical and serum analysis].

Drug treatments. The doses of psoralen and rhBMP-2 were selected based on previous studies $(20,22)$. Psoralen and rhBMP-2 were purchased from Yongjian Pharmaceutical Co. (Taizhou, China) and Amylet Scientific (PeproTech, Inc., Rocky Hill, NJ, USA), respectively. Psoralen powder was ground and suspended in physiological saline to achieve a final concentration of $4 \mathrm{mg} / \mathrm{ml}$. rhBMP-2 protein $(2.5 \mu \mathrm{g})$ was reconstituted with $50 \mu \mathrm{l}$ PBS to produce a $50 \mu \mathrm{g} / \mathrm{ml}$ solution. The rhBMP-2 solution was soaked onto a $4 \times 4 \times 5 \mathrm{~mm}$-sized absorbable collagen sponge (ACS; type-I collagen derived from bovine tendon; MEDECHI Medical Group, Shanghai, China) for a minimum of 15 min prior to implantation. A single implant was placed through the soft-tissue wound as an onlay bridging the fracture site. The mice were administered psoralen or physiological saline by oral gavage at a dose of $20 \mathrm{mg} / \mathrm{kg}$ per day from the first post-operative day ( $\mathrm{n}=10$ in each group).

Surgical protocol. All surgical equipment was sterilized in an autoclave. Sterile gowns, gloves, surgical masks and theater caps were used. Mice were anesthetized by intraperitoneal injection of ketamine hydrochloride (100 mg/ $\mathrm{kg}$ body weight) and xylazine ( $4 \mathrm{mg} / \mathrm{kg}$ body weight). A standardized fracture was created on the mid-diaphysis of the left femur in each mouse and stabilized with marrow-nailing. Next, an ACS carrier saturated with $2.5 \mu \mathrm{g}$ rhBMP-2 or PBS alone was inserted into the fracture. The muscles were subsequently repositioned and the skin was closed with suture. All of the animals received an intramuscular antibiotic and analgesic injection for 3 post-operative days. Unrestricted activity was allowed after the mice woke up from anesthesia.

Micro-CT examination. On day 21, five specimens per group were scanned by a Scanco MicroCT 60 system (45 kVp; 114 mA; Scanco Medical, Basserdorf, Switzerland) using a $12-\mu \mathrm{m}$ voxel size. The intramedullary pin was removed prior to scanning. In the callus analysis, the original cortical bone was excluded, and region of interest contained only new callus tissue. Mineralized tissue was distinguished from air or soft tissue at a fixed, global threshold with the lower limit corresponding to a mineral density of $421 \mathrm{mg}$ hydroxyapatite $/ \mathrm{cm}^{3}$ and the upper limit corresponding to $3,000 \mathrm{mg}$ hydroxyapatite $/ \mathrm{cm}^{3}$ (23). Total callus volume (TV), mineralized callus volume (BV), and tissue mineral density (TMD) were measured, and fraction of mineralized callus (BV/TV) was calculated as previously described (24).

Histology. For histological assessments, specimens from 5 animals per group were harvested on day 21 after fracture and fixed in $10 \%$ formalin for $24 \mathrm{~h}$, followed by decalcification in $10 \%$ EDTA for 2 weeks at room temperature. The specimens were then embedded in paraffin and cut longitudinally. Sagittal sections $(5 \mu \mathrm{m})$ were stained with H\&E for assessment of basic morphology and with Masson's trichrome for analysis of bone and cartilage using a standard histological staining protocol $(25,26)$.

Mechanical testing. The mechanical properties of the femurs with fracture harvested from 5 animals per group on day 21 post-surgery were assessed using a three-point bending test. Prior to mechanical testing, the femurs that had been preserved in a fridge $\left(4^{\circ} \mathrm{C}\right)$ for $12 \mathrm{~h}$ were warmed overnight at $22^{\circ} \mathrm{C}$. A material test machine (Instron-8841; Instron Corp., Norwood, MA, USA) with a $25 \mathrm{~N}$ load cell was used to test the femur to failure. The femurs were positioned horizontally with the anterior surface facing upwards, centered on the supports $10 \mathrm{~mm}$ 
Table I. Animal groups and interventions.

\begin{tabular}{lll}
\hline Group & $\mathrm{N}$ & \multicolumn{1}{c}{ Intervention } \\
\hline Model & 10 & Fracture, local implant of ACS + oral gavages of saline \\
rhBMP-2 & 10 & Fracture, local implant of ACS loaded with $2.5 \mu \mathrm{g}$ rhBMP-2 and oral gavages of saline \\
Psoralen + rhBMP-2 & 10 & Fracture, local implant of ACS loaded with $2.5 \mu \mathrm{g}$ rhBMP-2 and oral gavages of psoralen
\end{tabular}

rhBMP-2, recombinant human bone morphogenetic protein 2; ACS, absorbable collagen sponge.

A

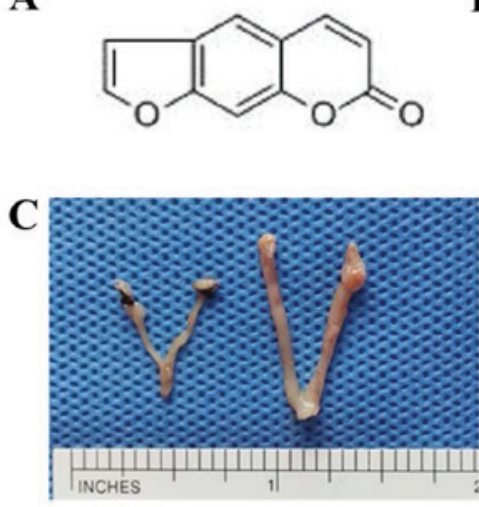

D

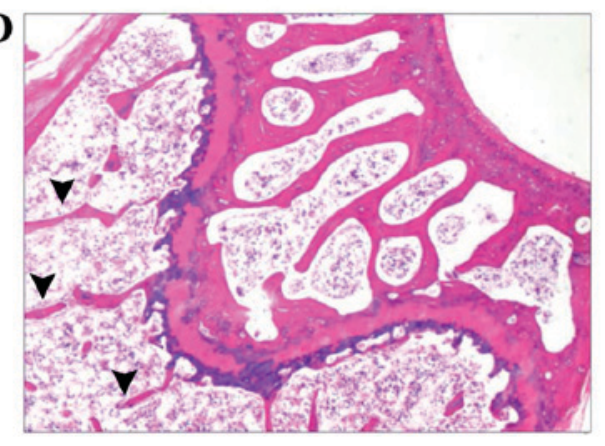

B

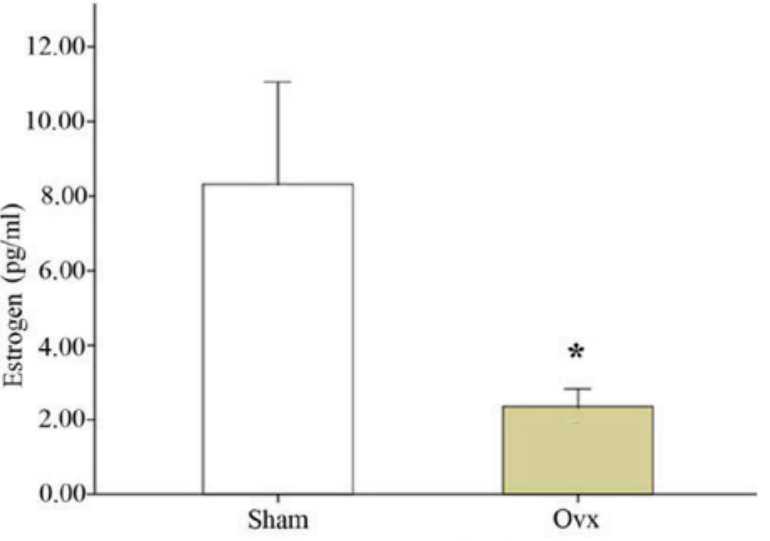

$\mathbf{E}$

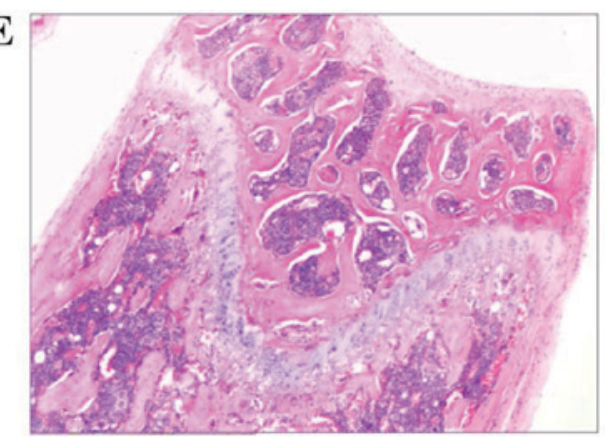

Figure 1. (A) Chemical structure of psoralen. (B) Bilateral OVX led to a significant decrease in estrogen levels compared with that in the sham mice. ${ }^{*} \mathrm{P}<0.05$ vs. sham. (C) Effect of decreased estrogen levels on bone growth and structure as exemplified by gonadal hypotrophy in OVX mice (left) compared with control mice (right). (D) Bone loss and trabecular bone deterioration (arrow) were evident in the OVX group (magnification, $\mathrm{x} 40$; H\&E staining). (E) Femoral metaphysis in the sham-operated mice (magnification, $\mathrm{x} 40$; H\&E staining). OVX, ovariectomy.

apart. A constant load was applied at the fracture center with a displacement rate of $5 \mathrm{~mm} / \mathrm{min}$ and directed vertically towards the mid-shaft with the anterior surface facing upward. After failure, the load vs. displacement curves were recorded. The maximum load and stiffness were calculated from the load-deflection curve recorded by a connected computer.

Serum analysis. On day 21 after fracture, blood (1 ml) was drawn from each of 5 animals per group by cardiac puncture under isoflurane anesthesia. Following standing in $2 \mathrm{ml}$ centrifuge tubes on ice for $10 \mathrm{~min}$, samples were centrifuged to separate the serum $\left(10,000 \mathrm{x}\right.$ g for $10 \mathrm{~min}$ at $\left.4^{\circ} \mathrm{C}\right)$, which was stored at $-80^{\circ} \mathrm{C}$. The serum samples were analyzed for BALP (cat. no. SEB091Mu), CTX-1 (cat. no. CEA665Mu) using ELISA kits from Cloud-Clone Co., Ltd. (Wuhan, China). The serum samples were also analyzed for estrogen using Cobas 6000 e601 Immunology analyzer (Roche Diagnostics GmbH, Mannheim, Germany).
Statistical analyses. Values are expressed as the mean \pm standard deviation. All statistical comparisons were performed using SPSS 17.0 software for Windows (SPSS, Inc., Chicago, IL, USA). Data were analyzed by one-way analysis of variance with a least-significant difference post-hoc test. Differences between means were considered statistically significant at the $5 \%$ confidence level $(\mathrm{P}<0.05)$.

\section{Results}

Confirmation of osteoporosis. The bilateral OVX led to a significant decrease in estrogen levels compared with those measured in sham mice $(\mathrm{P}<0.05$; Fig. 1B). The effects of decreased estrogen levels were also observed to result in gonadal hypotrophy in OVX mice (Fig. 1C). Compared with sham mice, the bone trabeculae of mice in the OVX group were arranged sparsely or had disappeared and indicated significant resorption (Fig. 1D and E). These results 

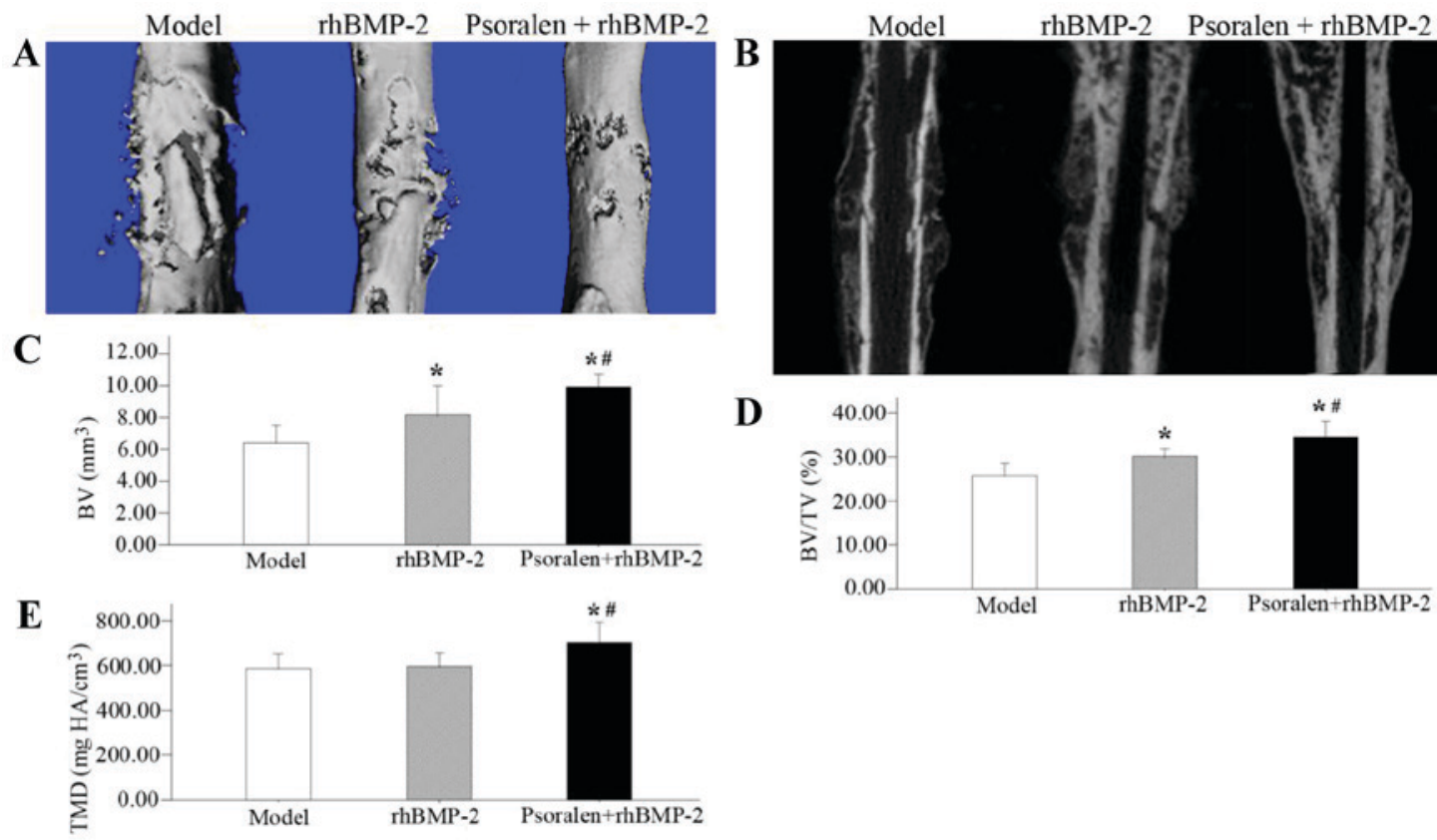

Figure 2. Micro-CT analysis. Representative (A) 3-dimensional reconstructions and (B) 2-dimensional coronal slices of the fractured mouse femurs obtained at 21 days after surgery. The images display $15-\mathrm{mm}$ sections of the diaphyses containing the fracture. Obvious differences in the internal and external structure of the calluses are present. (C-E) Graphical analysis of micro-CT measurements of fractured calluses. (C) BV, (D) BV/TV and (E) TMD. Values are expressed as the mean \pm standard deviation. ${ }^{*} \mathrm{P}<0.05$ vs. model group; ${ }^{\#} \mathrm{P}<0.05$ vs. rhBMP-2 group. BV/TV, ratio of mineralized callus volume vs. total callus volume; TMD, tissue mineral density; rhBMP-2, recombinant human bone morphogenetic protein 2; CT, computed tomography; BV, volume of mineralized callus.

confirmed the successful establishment of osteoporosis in the OVX mice.

Micro-CT examination. On day 21, the Micro-CT surface analyses indicated that fracture bridging occurred in the rhBMP-2 group and the Psoralen + rhBMP-2 group. Compared with the rhBMP-2 group, the healed bones in the Psoralen + rhBMP-2 group exhibited a near-physiological shape. In the model group, the fracture healed inadequately and at the same time, a large periosteal callus remained (Fig. 2A and B).

The quantitative results revealed that compared with the model group, the Psoralen + rhBMP-2 group had a higher BV $(\mathrm{P}=0.001), \mathrm{BV} / \mathrm{TV}(\mathrm{P}=0.001)$ and TMD $(\mathrm{P}=0.010)$, while the rhBMP-2 group exhibited an increase in $\mathrm{BV}(\mathrm{P}=0.022)$ and $\mathrm{BV} / \mathrm{TV}(\mathrm{P}=0.008)$, but no difference in TMD $(\mathrm{P}=0.814)$. Comparing the two treatment groups, the Psoralen + rhBMP-2 grouphad asignificantly greater $\mathrm{BV}(\mathrm{P}=0.024), \mathrm{BV} / \mathrm{TV}(\mathrm{P}=0.009)$ and TMD $(\mathrm{P}=0.015)$ than the rhBMP-2 group (Fig. 2C-E).

Histology. Representative histological images of fractures on day 21 are presented in Fig. 3. The calluses of all groups were composed of fibroblasts, cartilage and newly formed trabecular bone. Histological analysis of the model group samples indicated abundant amounts of chondroid callus fracture area and the formation of immature bone (Fig. 3A). The rhBMP-2 and Psoralen + rhBMP-2 groups exhibited progressive mineralized callus formation compared with the model group. However, the Psoralen + rhBMP-2 group displayed significantly more mineralized and mature callus compared with the rhBMP-2 group (Fig. 3B and C). Fig. 4A-C displays the osteoclasts in the model group, rhBMP-2 group and Psoralen + rhBMP-2 group, respectively, which were located in close association with the bone surface and appeared to be actively absorbing bone. In the Psoralen + rhBMP-2 group, osteoclasts were comparatively more sparse, but osteoclasts had a similar shape in all groups.

Mechanical testing. At 21 days after fracture, the data from the different treatment groups were compared with those of the model group. Averaged data regarding the biomechanical properties of the fractured femurs in each group are presented in Fig. 5. The highest values for maximum load, maximum stress and the flexure modulus were seen in the Psoralen + rhBMP-2 group. Compared with the model group, the psoralen + rhBMP-2 group had a significantly higher maximum load ( $\mathrm{P}=0.001$; Fig. $5 \mathrm{~A})$, maximum stress $(\mathrm{P}=0.004$; Fig. 5B) and the flexure modulus ( $\mathrm{P}=0.014$; Fig. 5C), while the rhBMP-2 group only exhibited improved in maximum stress $(\mathrm{P}=0.036)$. The maximum load $(\mathrm{P}=0.014)$ and flexure modulus $(\mathrm{P}=0.021)$ in the Psoralen + rhBMP-2 group were significantly higher compared with those in the rhBMP-2 group.

Serum analysis. On day 21, the serum levels of BALP and CTX-1 were measured to provide an evaluation of bone formation and resorption activity after fracture under psoralen and/or rhBMP-2 treatment (Fig. 6). BALP, a bone formation marker, was significantly increased in the Psoralen + rhBMP-2 group compared with that in the model group ( $\mathrm{P}=0.004$; Fig. 6A). BALP was also slightly increased in the rhBMP-2 group, but the difference was not statistically significant compared with the model group $(\mathrm{P}=0.667)$. Compared with that in the model group and the rhBMP-2 group, the serum levels of CTX-1, a bone resorption marker, were significantly decreased in the Psoralen + rhBMP-2 group $(\mathrm{P}=0.030$ and 0.021 , respectively; Fig. 6B). Regarding estrogen levels, no differences were detected among the groups (Fig. 6C). 


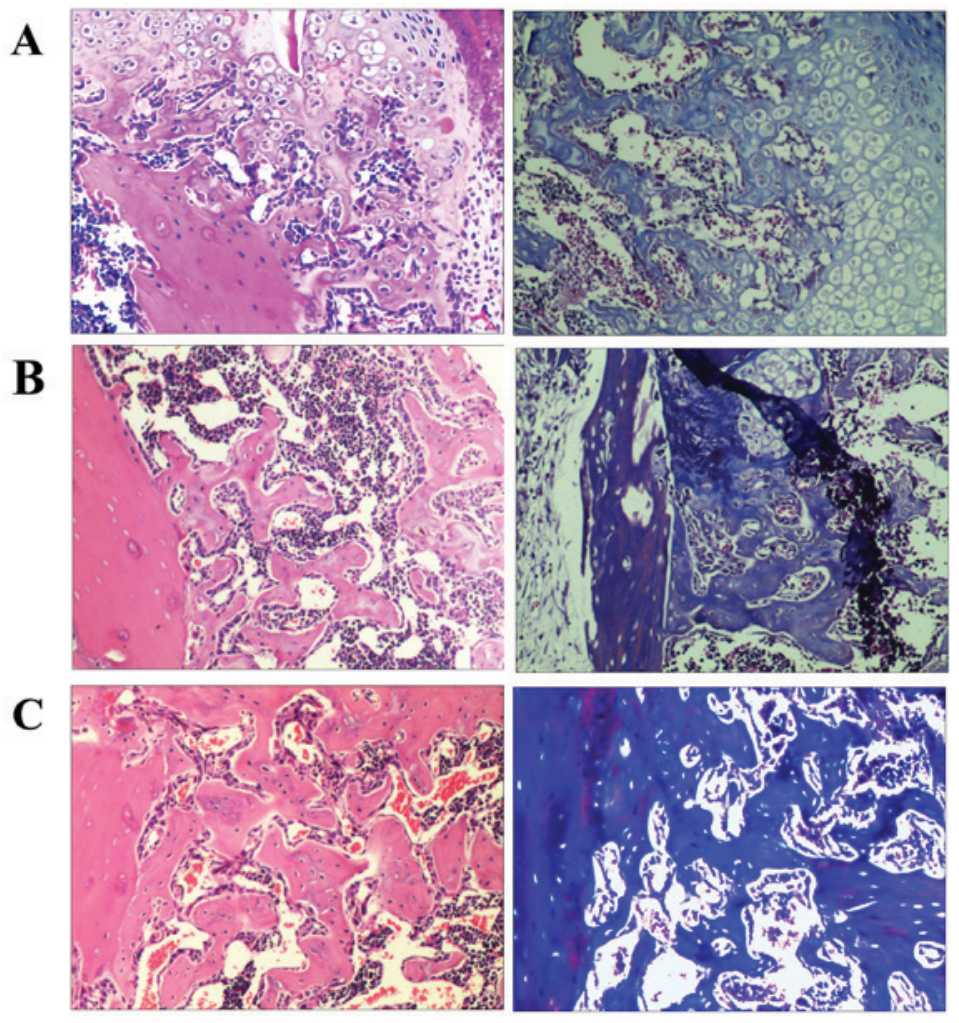

Figure 3. Histological analysis. Left and right columns display H\&E-and Masson's trichrome-stained micrographs, respectively, (magnification, x100) of fracture gaps in (A) the model group, (B) the rhBMP-2 group and (C) the Psoralen + rhBMP-2 group. rhBMP-2, recombinant human bone morphogenetic protein 2.

A

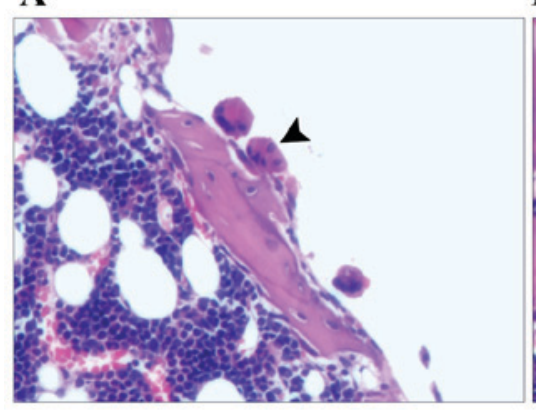

B

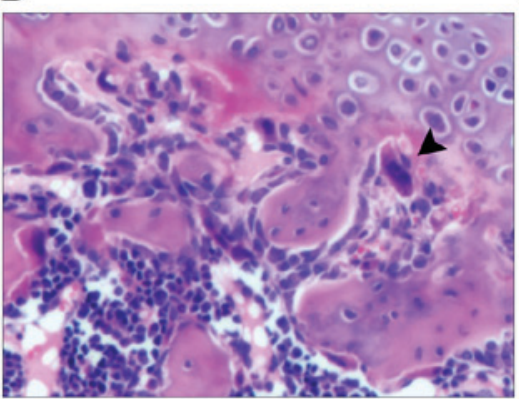

C

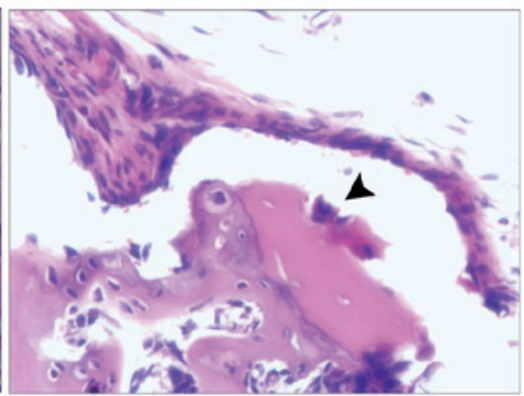

Figure 4. Histological sections (magnification, $\mathrm{x} 400$; H\&E staining) of osteoclasts (arrows). (A) Model group, (B) rhBMP-2 group and (C) Psoralen + rhBMP-2 group. rhBMP-2, recombinant human bone morphogenetic protein 2 .

\section{Discussion}

The present study demonstrated that in OVX mice, combined treatment with psoralen + rhBMP-2 had more potent effects on fracture healing than rhBMP-2 alone, as demonstrated by radiological, histological and mechanical analyses, with the highest values in micro-CT and mechanical parameters. These results indicate that orally administered psoralen and locally supplied rhBMP-2 had an additive effect on fracture healing in osteoporotic mice.

In the present study, at 6 weeks after the bilateral OVX, significant decreases in estrogen levels and uterine size were identified. Histological analysis of the distal femurs indicated that the trabecular bone almost disappeared near the growth plate in the OVX model, making it feasible to examine fracture healing in osteoporotic mice. Fracture healing is a multi-stage repair process that involves complex yet well-orchestrated steps that are initiated in response to injury, eventually resulting in the repair and restoration of function. Although the exact mechanism of impaired bone healing in post-menopausal osteoporosis remains to be fully elucidated, clinical studies and animal experiments have consistently indicated that fracture healing is impaired and delayed in osteoporotic bone $(27,28)$. As the aging population is expected to double by 2050 and the occurrence of osteoporotic fractures is to rise in the near future, impairment in osteoporotic fracture healing is becoming an emerging public health concern.

In contrast to conventional anti-resorptive drugs, bone-anabolic drugs build up new bone, which results in a faster increase of bone mass and strength. There is a great requirement for additional and affordable anabolic treatments to remedy impaired fracture healing. The evidence supporting 
A

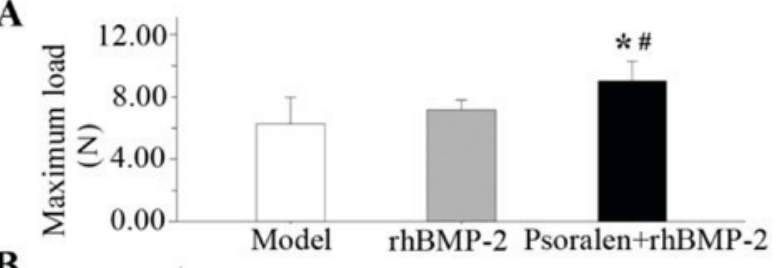

B

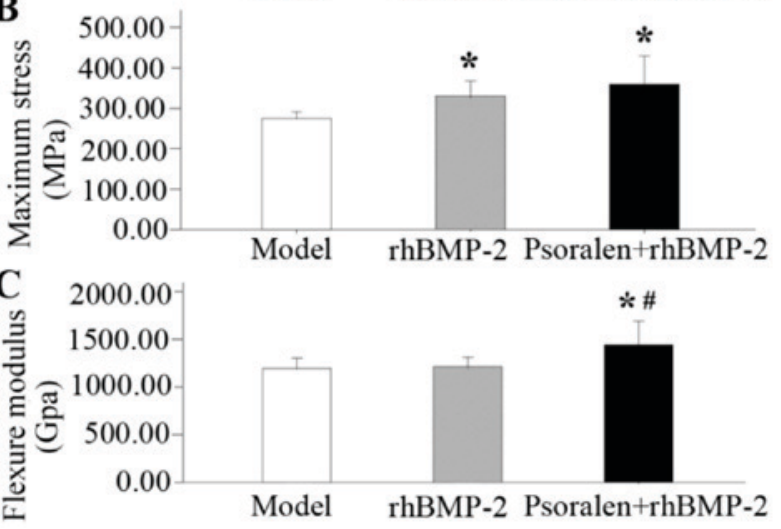

Figure 5. The fractured calluses of the model, rhBMP-2 and Psoralen + rhBMP-2 groups were subjected to 3-point bending analysis at 21 days of healing. (A) Maximum load, (B) maximum stress and (C) flexure modulus. Values are expressed as the mean \pm standard deviation. " $\mathrm{P}<0.05$ vs. model group; ${ }^{*} \mathrm{P}<0.05$ vs. rhBMP-2 group. rhBMP-2, recombinant human bone morphogenetic protein 2 .

the close association of BMPs with bone metabolism and the identification of the role of BMPs in the process of fracture healing and the pathogenesis of osteoporosis makes them attractive target molecules for the development of anabolic therapies for the prevention as well as for the treatment of osteoporotic fractures $(29,30)$. Consistent with other studies, which have reported enhanced fracture repair and stimulated early new bone formation with rhBMP-2 treatment in ovariectomized rats $(7,8)$, the present study indicated that rhBMP-2 has a positive effect on fracture healing in estrogen-deficient mice.

Certain medications are approved for the treatment of osteoporosis, including bisphosphonates, denosumab and estrogen replacement therapy; however, their effects on fracture healing in osteoporosis are limited and controversial. For instance, bisphosphonates, a major type of anti-osteoporosis medication used in the clinic, have negative effects of excessive suppression of physiological bone turnover, which results in inhibition of the bone remodeling process and impair of fracture healing (31). Certain studies even suggest that suspension of bisphosphonate use should be considered during the fracture healing period $(32,33)$. As an alternative treatment for osteoporosis and numerous other diseases, the therapeutic effects of natural products derived from plants have become an attractive research topic $(34,35)$.

Previous study has indicated that psoralen acts via activating BMP signaling to promote osteoblast differentiation (36). In the present study, psoralen enhanced anabolic bone formation caused by rhBMP-2. The micro CT imaging and histological analysis demonstrated that the group treated with a combination of oral psoralen and local rhBMP-2 produced more mature mineralized callus than local therapy with rhBMP-2 alone in OVX mice. Furthermore,
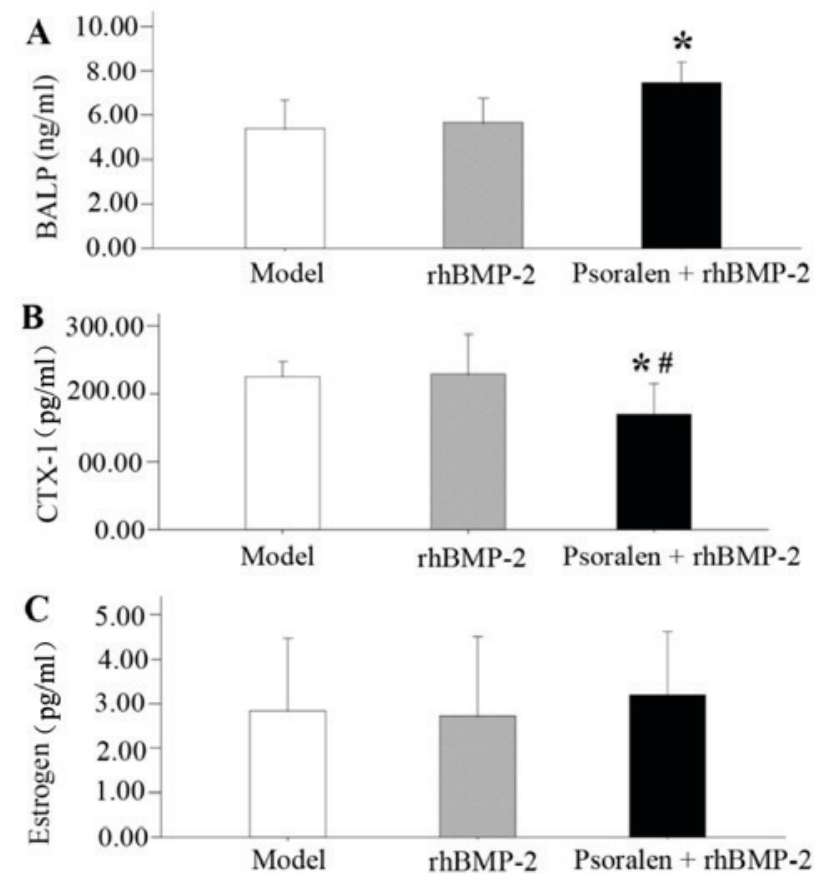

Figure 6. Serum analysis of (A) BALP, (B) CTX-1 and (C) estrogen. Values are expressed as the mean \pm standard deviation. ${ }^{*} \mathrm{P}<0.05$ vs. model group; ${ }^{\#} \mathrm{P}<0.05$ vs. rhBMP-2 group. rhBMP-2, recombinant human bone morphogenetic protein 2; BALP, bone-specific alkaline phosphatase; CTX-1, C-terminal telopeptide of type-1 collagen.

the biomechanical evaluation supported the results of the radiological and histological analyses. The highest biomechanical stability was measured in the group treated with psoralen + rhBMP-2. One reason for this may be that the systemic administration of psoralen and the local application of rhBMP-2 increased the local BMP levels to thereby have an additive effect on fracture healing in OVX mice.

In the present study, systemic administration of psoralen in the Psoralen + rhBMP-2 group exerted a dual regulatory effect to mediate osteoblast-osteoclast interactions. The serum analysis revealed that co-administration of psoralen not only increased the BALP levels, but also decreased the OVX-induced CTX-1 levels in the Psoralen + rhBMP-2 group. The histological analysis also revealed a decreased amount of osteoclasts in the Psoralen + rhBMP-2 group. It has been reported that BMPs indirectly stimulate osteoclastogenesis through osteoblasts via the receptor activator of nuclear factor $\kappa-\mathrm{B}$ (RANK)/RANK ligand (RANKL) pathway, which may lead to the premature resorption of BMP-induced bone $(37,38)$. A preclinical trial reported that BMP-2 caused initial resorption when placed in the metaphysis in a non-human primate core defect model in which bisphosphonates were successfully used to prevent the unwanted catabolic phase induced by BMP-2 (39). Zhang et al (40) have demonstrated that psoralen markedly inhibits the differentiation of osteoclasts by enhancing the expression of estrogen receptor and suppressing that of IL-17R. In another in vivo study, psoralen was reported to prevent osteoporosis via increasing osteoporogeterin expression and reducing RANKL levels to suppress osteoclast differentiation and maturity (41). This may provide an explanation for the results obtained in the present study, indicating that daily systemic 
administration of psoralen may reduce bone resorption produced by increased activity of osteoclasts caused by rhBMP-2.

No matter how complex the mechanisms of action may be, the phytoestrogens in psoralen may have an important role in the amelioration of post-menopausal bone loss. To determine whether psoralen behaved in a similar manner to estradiol in OVX mice, the estrogen levels in the serum were determined. The results indicated that treatment of OVX mice with psoralen did not significantly improve the estrogen levels compared with those in the model group, therefore further studies are needed to investigate the effect of psoralen on the uterus.

Of note, the present study had several limitations. First, the animals were euthanized on day 21 , and therefore it was not possible to determine the long-term influence of the treatments on bone remodeling. Second, given the saturation of psoralene in water, some of the suspended powder may have stuck to the syringe. The effective dose of gavage may be lower than that expected in the experimental design. Furthermore, only one dose of psoralen was assessed in the present study, and the observation of the healing in the psoralen group may have been better if a range of psoralen doses had been tested. In addition, further studies are required to confirm whether there is indeed a difference in the local BMPs levels among the groups. Finally, the specific molecular mechanisms of the synergistic effects of psoralen and rhBMP-2 in promoting osteogenesis are elusive and require further study.

In conclusion, psoralen + rhBMP-2 in combination produced better fracture healing than rhBMP-2 in mice with OVX-induced osteoporosis. While rhBMP-2 is a costly substance, psoralen, as an active component from natural herbs, is inexpensive, has been approved worldwide, is well-tolerated and has few side effects. Combining a local osteo-inductive agent with a systemic anabolic agent may be a novel paradigm in the treatment of osteoporotic fracture.

\section{Acknowledgements}

We acknowledge the support received from Professor Kebin Liu (Department of Orthopaedics, The First Affiliated Hospital of Yangtze University, Jingzhou, China) for proofreading the manuscript.

\section{Funding}

The present study was financially supported by the Natural Science Foundation of Hubei Province (grant no. 2012FFB03601) and the Science and Technology Development Plan Project of Jingzhou City (grant no. 2017041).

\section{Availability of data and materials}

The datasets used and/or analyzed during the current study are available from the corresponding author on reasonable request.

\section{Authors' contributions}

$\mathrm{KH}$ contributed to the conception of the study and wrote the manuscript. JZ contributed significantly to the statistical analyses and the writing of the manuscript. GW and $\mathrm{KH}$ performed the experiments and data analyses. SP helped to design the experiments, interpret the data and perform the analysis with constructive discussions. The final version of the manuscript has been read and approved by all authors, and each author believes that the manuscript represents honest work.

\section{Ethics approval and consent to participate}

The Animal experimentation ethics committee of Yangtze University (Jingzhou, China) approved the experimental animal protocol of the present study.

\section{Patient consent for publication}

Not applicable.

\section{Competing interests}

The authors declare that they have no competing interests.

\section{References}

1. Broderick JM, Bruce-Brand R, Stanley E and Mulhall KJ: Osteoporotic hip fractures: The burden of fixation failure. ScientificWorldJournal 2013: 515197, 2013.

2. Singer A, Exuzides A, Spangler L, O'Malley C, Colby C, Johnston K, Agodoa I, Baker J and Kagan R: Burden of illness for osteoporotic fractures compared with other serious diseases among postmenopausal women in the United States. Mayo Clin Proc 90: 53-62, 2015.

3. McCann RM, Colleary G, Geddis C, Clarke SA, Jordan GR, Dickson GR and Marsh D: Effect of osteoporosis on bone mineral density and fracture repair in a rat femoral fracture model. J Orthop Res 26: 384-393, 2008.

4. Goldhahn J, Féron JM, Kanis J, Papapoulos S, Reginster JY, Rizzoli R, Dere W, Mitlak B, Tsouderos Y and Boonen S: Implications for fracture healing of current and new osteoporosis treatments: An ESCEO consensus paper. Calcif Tissue Int 90: 343-353, 2012.

5. Watson JT and Nicolaou DA: Orthobiologics in the augmentation of osteoporotic fractures. Curr Osteoporos Rep 13: 22-29, 2015.

6. U.S. Food and Drug Administration. InFuse TM Bone Graft/LT-CAGETM lumbar tapered fusion device-P000058, 2002. Available at: https://www.accessdata.fda.gov/scripts/ cdrh/cfdocs/cfpma/pma.cfm?id=P000058. Accessed January 23, 2014.

7. Sarban S, Senkoylu A, Isikan UE, Korkusuz P and Korkusuz F: Can rhBMP-2 containing collagen sponges enhance bone repair in ovariectomized rats? A preliminary study. Clin Orthop Relat Res 467: 3113-3120, 2009.

8. Park SB, Park SH, Kim NH and Chung CK: BMP-2 induced early bone formation in spine fusion using rat ovariectomy osteoporosis model. Spine J 13: 1273-1280, 2013.

9. Zarrinkalam MR, Schultz CG, Ardern DW, Vernon-Roberts B and Moore RJ: Recombinant human bone morphogenetic protein-type 2 (rhBMP-2) enhances local bone formation in the lumbar spine of osteoporotic sheep. J Orthop Res 31: 1390-1397, 2013.

10. Boden SD, Kang J, Sandhu H and Heller JG: Use of recombinant human bone morphogenetic protein-2 to achieve posterolateral lumbar spine fusion in humans: A prospective, randomized clinical pilot trial: 2002 volvo award in clinical studies. Spine (Phila Pa 1976) 27: 2662-2673, 2002.

11. Hurlbert RJ, Alexander D, Bailey S, Mahood J, Abraham E, McBroom R, Jodoin A and Fisher C: Rhbmp-2 for posterolateral instrumented lumbar fusion: A multicenter prospective randomized controlled trial. Spine (Phila $\mathrm{Pa}$ 1976) 38: 2139-2148, 2013. 
12. Bess S, Line BG, Lafage V, Schwab F, Shaffrey CI, Hart RA, Boachie-Adjei O, Akbarnia BA, Ames CP, Burton DC, et al: Does recombinant human bone morphogenetic protein-2 use in adult spinal deformity increase complications and are complications associated with location of rhBMP-2 use? A prospective, multicenter study of 279 consecutive patients. Spine (Phila Pa 1976) 39: 233-242, 2014.

13. Simmonds MC, Brown JV, Heirs MK, Higgins JP, Mannion RJ, Rodgers MA and Stewart LA: Safety and effectiveness of recombinant human bone morphogenetic protein-2 for spinal fusion: A meta-analysis of individual-participant data. Ann Intern Med 158: 877-889, 2013.

14. Faundez A, Tournier C, Garcia M, Aunoble S and Le Huec JC: Bone morphogenetic protein use in spine surgery-complications and outcomes: A systematic review. Int Orthop 40: 1309-1319, 2016.

15. Kyllönen L, D'Este M, Alini M and Eglin D: Local drug delivery for enhancing fracture healing in osteoporotic bone. Acta Biomater 11: 412-434, 2015.

16. Yu NY, Gdalevitch M, Murphy CM, Mikulec K, Peacock L, Fitzpatrick J, Cantrill LC, Ruys AJ, Cooper-White JJ, Little DG and Schindeler A: Spatial control of bone formation using a porous polymer scaffold co-delivering anabolic rhBMP-2 and anti-resorptive agents. Eur Cell Mater 27: 98-109, 2014.

17. Larsson S and Fazzalari NL: Anti-osteoporosis therapy and fracture healing. Arch Orthop Trauma Surg 134: 291-297, 2014.

18. Hegde V, Jo JE, Andreopoulou P and Lane JM: Effect of osteoporosis medications on fracture healing. Osteoporos Int 27: 861-871, 2016.

19. Yang Z, Huang JH, Liu SF, Zhao YJ, Shen ZY, Wang YJ and Bian Q: The osteoprotective effect of psoralen in ovariectomy-induced osteoporotic rats via stimulating the osteoblastic differentiation from bone mesenchymal stem cells. Menopause 19: 1156-1164, 2012.

20. Yuan X, Bi Y, Yan Z, Pu Q, Li Y and Zhou K: Psoralen and isopsoralen ameliorate sex hormone deficiency-induced osteoporosis in female and male mice. Biomed Res Int 2016: 6869452, 2016.

21. Manigrasso MB and O'Connor JP: Comparison of fracture healing among different inbred mouse strains. Calcif Tissue Int 82: 465-474, 2008.

22. Murnaghan M, Mcllmurray L, Mushipe MT and Li G: Time for treating bone fracture using rhBMP-2: A randomised placebo controlled mouse fracture trial. J Orthop Res 23: 625-631, 2005.

23. Toupadakis CA, Granick JL, Sagy M, Wong A, Ghassemi E, Chung DJ, Borjesson DL and Yellowley CE: mobilization of endogenous stem cell populations enhances fracture healing in a murine femoral fracture model. Cytotherapy 15: 1136-1147, 2013

24. Bouxsein ML, Boyd SK, Christiansen BA, Guldberg RE, Jepsen KJ and Müller R: Guidelines for assessment of bone microstructure in rodents using micro-computed tomography. J Bone Miner Res 25: 1468-1486, 2010.

25. Toğral G, Arikan M, Korkusuz P, Hesar RH and Ekșioğlu MF: Positive effect of tadalafil, a phosphodiesterase-5 inhibitor, on fracture healing in rat femur. Eklem Hastalik Cerrahisi 26: 137-144, 2015

26. Manigrasso MB and O'Connor JP: Characterization of a closed femur fracture model in mice. J Orthop Trauma 18: 687-695, 2004.
27. Cheung WH, Miclau T, Chow SK, Yang FF and Alt V: Fracture healing in osteoporotic bone. Injury 47 (Suppl): S21-S26, 2016.

28. Tarantino U, Cerocchi I, Scialdoni A, Saturnino L, Feola M, Celi M, Liuni FM, Iolascon G and Gasbarra E: Bone healing and osteoporosis. Aging Clin Exp Res 23: 62-64, 2011.

29. Kanakaris NK, Petsatodis G, Tagil M and Giannoudis PV: Is there a role for bone morphogenetic proteins in osteoporotic fractures? Injury 40 (Suppl): S21-S26, 2009.

30. Van Lieshout EM and Alt V: Bone graft substitutes and bone morphogenetic proteins for osteoporotic fractures: What is the evidence? Injury 47 (Suppl): S43-S46, 2016.

31. Girgis CM, Sher D and Seibel MJ: Atypical femoral fractures and bisphosphonate use. N Engl J Med 362: 1848-1849, 2010

32. Savaridas T, Wallace RJ, Salter DM and Simpson AH: Do bisphosphonates inhibit direct fracture healing?: A laboratory investigation using an animal model. Bone Joint J 95: 1263-1268, 2013.

33. Ha KY,Park KS, Kim SI and Kim YH: Does bisphosphonate-based anti-osteoporosis medication affect osteoporotic spinal fracture healing? Osteoporos Int 27: 483-488, 2016.

34. Leung PC and Siu WS: Herbal treatment for osteoporosis: A current review. J Tradit Complement Med 3: 82-87, 2013.

35. An J, Yang H, Zhang Q, Liu C, Zhao J, Zhang L and Chen B: Natural products for treatment of osteoporosis: The effects and mechanisms on promoting osteoblast-mediated bone formation. Life Sci 147: 46-58, 2016.

36. Tang DZ, Yang F, Yang Z, Huang J, Shi Q, Chen D and Wang YJ: Psoralen stimulates osteoblast differentiation through activation of bmp signaling. Biochem Biophys Res Commun 405: 256-261, 2011.

37. Kanatani M, Sugimoto T, Kaji H, Kobayashi T, Nishiyama K, Fukase M, Kumegawa M and Chihara K: Stimulatory effect of bone morphogenetic protein-2 on osteoclast-like cell formation and bone-resorbing activity. J Bone Miner Res 10: 1681-1690, 1995.

38. Okamoto M, Murai J, Yoshikawa $\mathrm{H}$ and Tsumaki N: Bone morphogenetic proteins in bone stimulate osteoclasts and osteoblasts during bone development. J Bone Miner Res 21: 1022-1033, 2006

39. Seeherman HJ, Li XJ, Gavin D, Wozney JM and Bouxsein ML: Bisphosphonate limits initial bone resorption without decreasing bone induction in rhBMP-2/ACS treated nonhuman primate core defects. Bone 30 (Suppl): 44S-44S, 2002.

40. Zhang WJ, Xie BP, Li WJ, Li JP, Gan GX and Zhang J: Inhibitory effect of psoralen on osteoclast formation and its underling mechanism in vitro. J Third Milit Med Univ 39: 641-645, 2017 (In Chinese)

41. Wang JH, Guo M, Zheng L and Wang Z: Effects of psoralen on osteoporogeterin and receptor activator nuclear factor kappa B ligand mRNA expression in rat osteoblasts. Zhongguo Zuzhi Gongcheng Yanjiu yu Linchuang Kangfu 14: 6927-6930, 2010 (In Chinese).

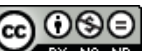

This work is licensed under a Creative Commons Attribution-NonCommercial-NoDerivatives 4.0 International (CC BY-NC-ND 4.0) License. 